\title{
Impact of the DC Cable Models on the SVD Analysis of a Multi-Terminal HVDC System
}

\author{
Samy Akkari*, Eduardo Prieto-Araujo ${ }^{\dagger}$, Jing Dai*, Oriol Gomis-Bellmunt ${ }^{\dagger}$ and Xavier Guillaud ${ }^{\ddagger}$ \\ * Group of Electrical Engineering Paris (GeePs) \\ CentraleSupélec, CNRS UMR 8507, Université Paris-Sud and Université Pierre et Marie Curie \\ $\dagger$ Centre d'Innovació Tecnològica en Convertidors Estàtics i Accionaments (CITCEA-UPC) \\ Universitat Politècnica de Catalunya, ETS d'Enginyeria Industrial de Barcelona \\ $\ddagger$ Laboratory of Electrical Engineering and Power Electronics (L2EP Lille) \\ Contact person: samy.akkariecentralesupelec.fr
}

\begin{abstract}
High Voltage Direct Current (HVDC) grids are complex Multi-Inputs Multi-Outputs (MIMO) systems whose dynamics are difficult to assess. This paper first describes the modelling of VSC-based Multi-Terminal HVDC systems (MTDC) using different existing cable models. It then recalls the benefits of the Singular Value Decomposition (SVD) approach for the frequency analysis of such systems, and describes how to perform an SVD analysis on a Multi-Terminal HVDC (MTDC) system from its state-space representation. The paper discusses the results of the SVD analysis with regards to the selection of the voltage-droop parameter and the DC voltage constraint of the DC grid. It then emphasises the impact of the choice of the DC cable model on the results of the SVD study and illustrates the cable model influence on the MTDC system through an ElectroMagnetic Transient (EMT) simulation.
\end{abstract}

Index Terms-Cable model, High Voltage Direct Current (HVDC), Multi-terminal HVDC (MTDC), Singular Value Decomposition (SVD), Voltage droop control

\section{INTRODUCTION}

The ever-growing number of commissioned offshore windfarms in the North Sea raised numerous technical challenges [1]. So far, the most feasible solution to transmit bulk power over long distances is to use High Voltage Direct Current (HVDC) technology. Besides, Voltage Source Converter (VSC-HVDC) is currently the most appropriate and mature technology to interconnect the wind farms and the mainland grids [2]. This will probably give rise to several DC links located in the same geographical zone; DC reinforcements, extensions and $\mathrm{AC}$ zone interconnections may then lead to a Multi-Terminal HVDC (MTDC) grid in the future.

The control of HVDC grids is mandatory to maintain the stability of the system. The master-slave command strategy of a DC link is not suitable for MTDC systems since the single AC bus connected to the master converter would have to provide all the balancing power and that the loss of this "master" converter would mean the loss of the whole system. To remedy this problem, the voltage droop control technique described in [3]-[6], where several VSCs participate in the DC voltage regulation at the same time, is probably the most adequate control strategy to safely and reliably operate any MTDC system.
However, in those references, the voltage-droop parameter is either selected from a static point of view, or simply arbitrarily chosen equal to 0.05 p.u./p.u. In this paper, a power-based droop control is designed to tolerate a maximum DC voltage deviation of the MTDC grid, both in steady state and during transients. This can be achieved by using the multi-variable frequency analysis based on the Singular Value Decomposition (SVD).

The SVD tool was first applied to MTDC systems to size the voltage-droop gain in [7], where the converters were modelled as simple current injectors with a current-based voltage droop and the DC lines by classical PI sections. Reference [8] then used the SVD tool with a different objective: to minimize the impact of AC-side disturbances. Finally, Reference [9] improved the work of [7] by including the dynamics of the control loops of the DC grid converters in the frequency analysis and thus in the voltage-droop gain selection. The difference between the results in [7] and [9] is not negligible, implying that the level of complexity of the component models, such as the control loops or the DC cable model, highly impacts the results of the SVD study and thus the design of the voltagedroop gain.

Since References [7]-[9] are, to the authors' knowledge, the only references that applied the SVD tools to HVDC systems, the basic principle of this method is recalled and further explained in this paper. Then, the SVD is used to size the voltage-droop parameter of the VSCs of an MTDC system. Finally, this article intends to show the impact of four basic DC cable models on the frequency response of the MTDC system obtained in the SVD analysis, and thus, on the voltage-droop gain selection. The different DC cable models are used in an EMT simulation in order to analyse their impact on the system in the time domain.

The paper is organised as follows. The next section describes the MTDC system and four DC cable models. Then Section III recalls the fundamentals of the Singular Value Decomposition method. Section IV justifies the use of the multi-variable approach in the design of the voltage-droop gain and illustrates the selection of the voltage-droop parameter with regard to a voltage constraint by using the SVD approach. The impact of the DC cable models on the singular value 
representation and on the EMT simulation of the system is then assessed in Section V. Finally, conclusions are drawn in Section VI.

\section{Voltage Source CONVERTERS AND CABle MODELLING}

\section{A. DC cable modelling}

Because of the numerous dynamics induced by the VSC control loops, particular attention must be paid to the frequency domain validity of the linear cable models used to compute the state-space representation of the MTDC system. In this section, four very basic models are presented and they will be used in later sections to assess their impact on the dynamics of the MTDC system. The DC cable parameters are listed in Appendix.

Since the DC capacitors have a strong impact on the DC voltage, the first DC cable model is simply a resistor-capacitor (RC) section as depicted in Figure 1(a). The second model is the classical PI section model used in [7] and [9] and depicted in Figure 1(b). The third DC cable model is basically PI section but with several parallel branches [10], as depicted in Figure 1(c), where the parallel branches have been calculated through a fitting algorithm to fit the frequency response of the frequency-dependent elements of a wide-band cable [11]. Finally, the fourth DC cable model is an improved PI section where the coupling between the core and the screen of the cable is represented by a mutual inductance between the two [6], [12], as pictured in Figure 1(d). This coupling generates a current that flows through the resistance of the screen, and since this resistance is significant, the transient state of the system is better damped overall than when the screen is totally neglected (as with a classical PI section).

\section{B. MTDC System Modelling}

The modelling of the VSCs, the control of the converters including their inner and outer control loops, and the acquisition of their state-space representations is described in [13]. The overall control strategy of the VSCs is described in Figure 2

The MTDC system considered in this paper is a 3-terminal HVDC system [9], as depicted in Figure 3. An offshore WindFarm Converter (WFC) is connected to two different Grid-Side Converters (GSC) by means of a 3-terminal meshed HVDC grid. The DC cable lengths are respectively $300 \mathrm{~km}$ between the GSC 1 and the WFC, and $200 \mathrm{~km}$ between the GSC 2 and the WFC. The procedure followed to obtain the state-space model of the system is described in [13].

As illustrated in Figure 2, the two GSCs are equipped with voltage-droop controllers (see [3]-[6]) such that they participate in the DC voltage regulation by adapting their power withdrawal from the DC grid according to the equation

$$
\Delta P_{v}=\frac{1}{k_{v}} \Delta u_{s}
$$

where $\Delta P_{v}$ is the reference value for the power deviation that is to be added to the actual power withdrawal from the DC

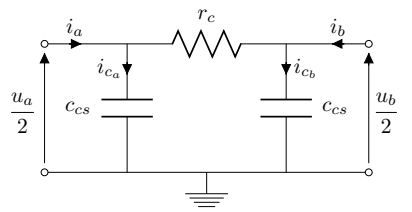

(a) Resistor-Capacitor (RC) section.

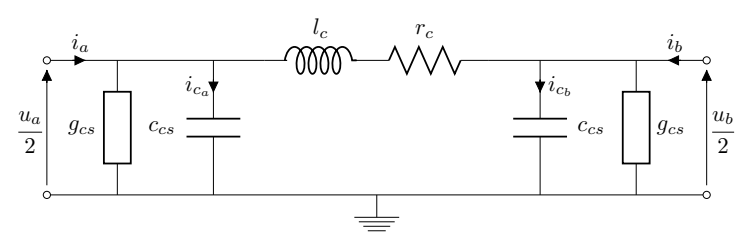

(b) Classical PI section.

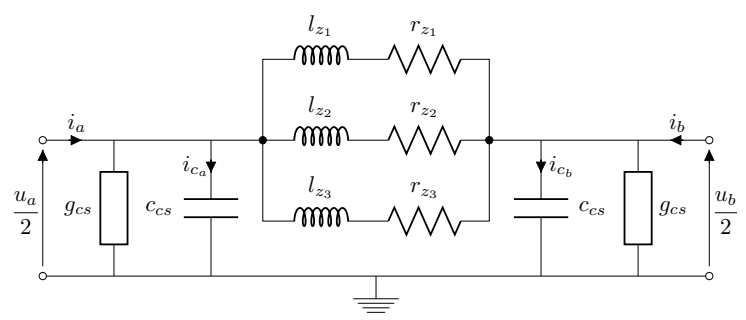

(c) "Fitted PI" section [10], where the parallel branches elements have been calculated through a fitting algorithm to fit the frequency response of the frequency-dependent elements of a wide-band cable [11].

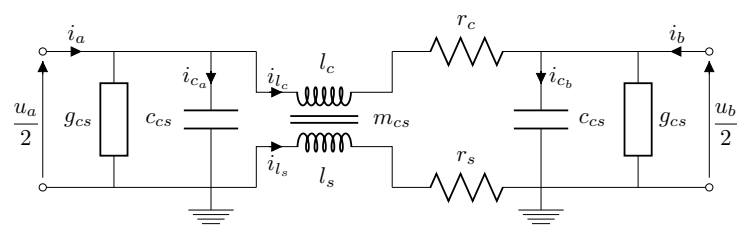

(d) "Coupled-PI" section, with a mutual inductance between the core and the screen [6], [12].

Figure 1. DC cable models.

grid $P_{g}^{*}, k_{v}$ is the voltage-droop parameter and $\Delta u_{s}$ is the DC voltage deviation at the converter's terminal.

The Wind-Farm Converter (WFC) is not equipped with this extra controller since its purpose is to inject all the available wind power into the HVDC grid.

\section{Singular Value Decomposition Method}

\section{A. Transfer Matrix of the Multi-Variable MTDC System}

Since the considered 3-terminal MTDC system is a MultiInput Multi-Output (MIMO) system, this study focuses on the evolution of the DC voltage deviation of the three stations (3 outputs: $\Delta u_{s_{1}}, \Delta u_{s_{2}}$ and $\Delta u_{s_{3}}$ ) when excited by the powerreference deviations of the converters (3 inputs: $\Delta P_{g_{1}}^{*}, \Delta P_{g_{2}}^{*}$ and $\Delta P_{g_{3}}^{*}$ ). Thus, as portrayed in Figure 4 , the basic transfer function model is $y(s)=G(s) u(s)$, where $y$ is a $3 \times 1$ output vector, $u$ is a $3 \times 1$ input vector and $G(s)$ is a $3 \times 3$ transfer function matrix.

The study aims at determining an optimal voltage-droop gain that constrains the DC voltage deviation of each converter 


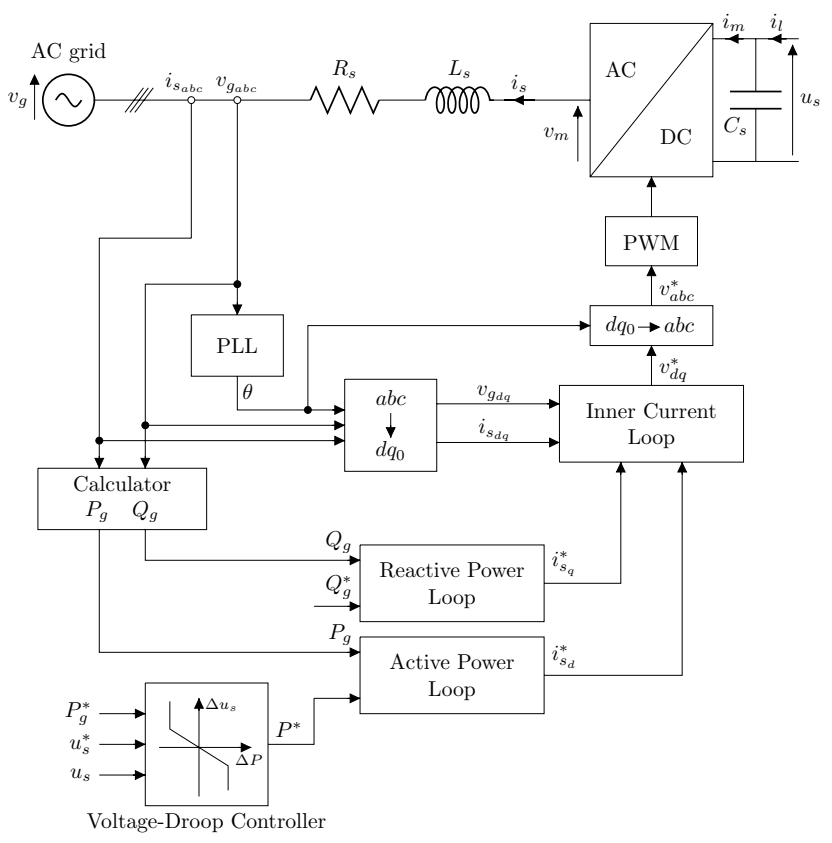

Figure 2. Control strategy of a VSC-HVDC converter.

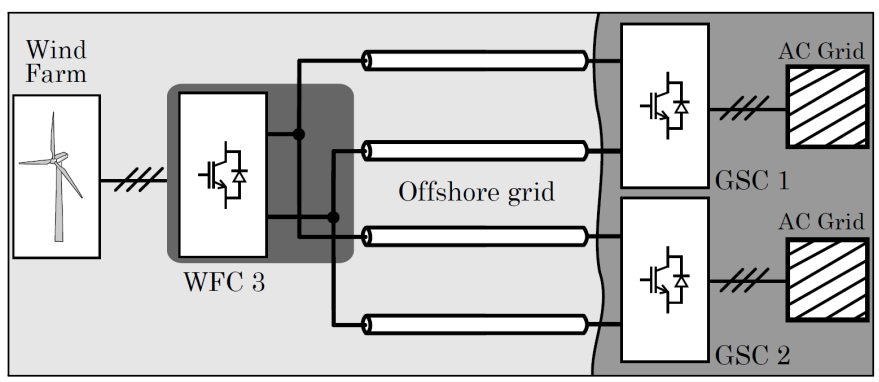

Figure 3. Three-terminal HVDC system. [9]

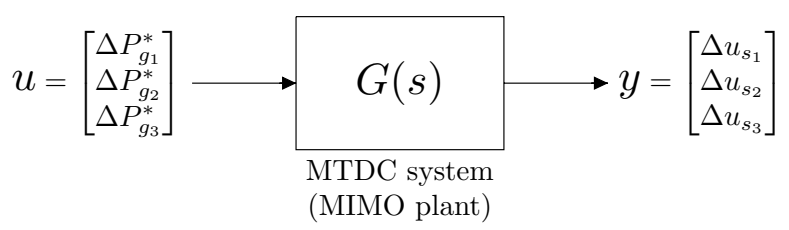

Figure 4. Transfer function model of the considered 3-terminal MTDC system.

station (the outputs) within a range of $\pm 5 \%$, for any powerreference change of the converters (the inputs), from both a static and a dynamic point of view.

As the analysed system is a MIMO system, a change in one power-reference of the converters, for example a powerreference deviation of the first GSC, $\Delta P_{g_{1}}^{*}$, affects the DC voltages of every station (the outputs $\Delta u_{s_{1}}, \Delta u_{s_{2}}$ and $\Delta u_{s_{3}}$ ). Hence, different power-reference deviation input vectors $u=$ $\left[\begin{array}{ccc}\Delta P_{g_{1}}^{*} & \Delta P_{g_{2}}^{*} & \Delta P_{g_{3}}^{*}\end{array}\right]^{T}$, even if they have the same norm, can have very distinct impacts on the DC voltage deviation output vectors $y=\left[\begin{array}{lll}\Delta u_{s_{1}} & \Delta u_{s_{2}} & \Delta u_{s_{3}}\end{array}\right]^{T}$. This illustrates the main difference between a scalar (SISO) system and a
MIMO system: the presence of directions in the latter that impacts the gain of the plant [14].

In fact, the gain matrix of the system $G(s)$ between a particular input signal $u(\omega)$ and the induced output $y(\omega)$ is given by, in terms of the $\mathrm{L}^{2}$ norm:

$$
\begin{aligned}
\frac{\|y(\omega)\|_{2}}{\|u(\omega)\|_{2}} & =\frac{\|G(j \omega) u(\omega)\|_{2}}{\|u(\omega)\|_{2}} \\
& =\frac{\sqrt{\Delta u_{s_{1}}^{2}+\Delta u_{s_{2}}^{2}+\Delta u_{s_{3}}^{2}}}{\sqrt{\Delta P_{g_{1}}^{* 2}+\Delta P_{g_{2}}^{* 2}+\Delta P_{g_{3}}^{* 2}}} \\
& =\frac{\sqrt{\sum_{j=1}^{3}\left(\sum_{k=1}^{3} g_{j k} \Delta P_{g_{k}}^{*}\right)^{2}}}{\sqrt{\Delta P_{g_{1}}^{* 2}+\Delta P_{g_{2}}^{* 2}+\Delta P_{g_{3}}^{* 2}}}
\end{aligned}
$$

where $g_{j k}$ is the gain of the system for the output $j$ in the direction of the input $k$.

Equation (2) shows that the gain of the system depends on the frequency $\omega$, and since the system is linear, it is independent of the input magnitude $\|u(\omega)\|_{2}$. However, the gain depends on the direction of the input $u$.

\section{B. Frequency Response Analysis of an MTDC System}

The singular value decomposition technique can be considered as an expansion of the Bode frequency representation for multi-variable systems, that takes into account the input and output directions of the system.

The singular values of the system transfer function matrix $G(j \omega)$ at the pulsation $\omega$ are given by:

$$
\sigma_{i}(G(j \omega))=\sqrt{\lambda_{i}\left(G^{T}(j \omega) G(j \omega)\right)}
$$

where $\lambda_{i}(\cdot)$ is the $i$-th eigenvalue of the matrix $G^{T} G$.

Since the gain depends on the direction of the input vector, which can significantly change with the pulsation $\omega$, the gain in different directions of the system at any pulsation $\omega$ must be considered when extracting the maximum gain among all the possible input vectors. The singular values obtained with (3) express the gain of each one of the input-directions in the output-directions of the system (see [14] for more details).

The largest gain for any input direction $u(\omega)$ at pulsation $\omega$ is then equal to the maximum singular value $\bar{\sigma}(G(j \omega))$ :

$$
\max _{u \neq 0} \frac{\|G(j \omega) u(\omega)\|_{2}}{\|u(\omega)\|_{2}} \triangleq \bar{\sigma}(G(j \omega))
$$

The maximum allowable voltage deviation and the maximum possible power-reference change of the converters can then be represented as a gain boundary in the multi-variable frequency response of the MTDC system and compared to the maximum of the singular values of the system. In fact, by ensuring that the maximum of the singular values does not bypass the gain boundaries corresponding to the $\mathrm{DC}$ voltage deviation, the MTDC system is assured to comply with the imposed constraint. 


\section{Comparison between the SVD and the Transfer Functions}

The SVD representation is a useful tool that allows the quantification of the maximum gain of the system, a task that can not be achieved by solely studying its transfer function. In fact, the individual study of all the transfer functions is not only heavy (as their number grows rapidly with that of inputs and outputs of the system), but also misleading since the gain of the MIMO system depends on the direction of the input vector, and hence this gain can not be assessed by studying separately the transfer function between each input-output pair of the system.

Figure 5 shows a comparison between the SVD representation and the 9 transfer functions of the 3-terminal MTDC system whose input and output vectors are respectively $u=$ $\left[\begin{array}{ccc}\Delta P_{g_{1}}^{*} & \Delta P_{g_{2}}^{*} & \Delta P_{g_{3}}^{*}\end{array}\right]^{T}$ and $y=\left[\begin{array}{lll}\Delta u_{s_{1}} & \Delta u_{s_{2}} & \Delta u_{s_{3}}\end{array}\right]^{T}$.

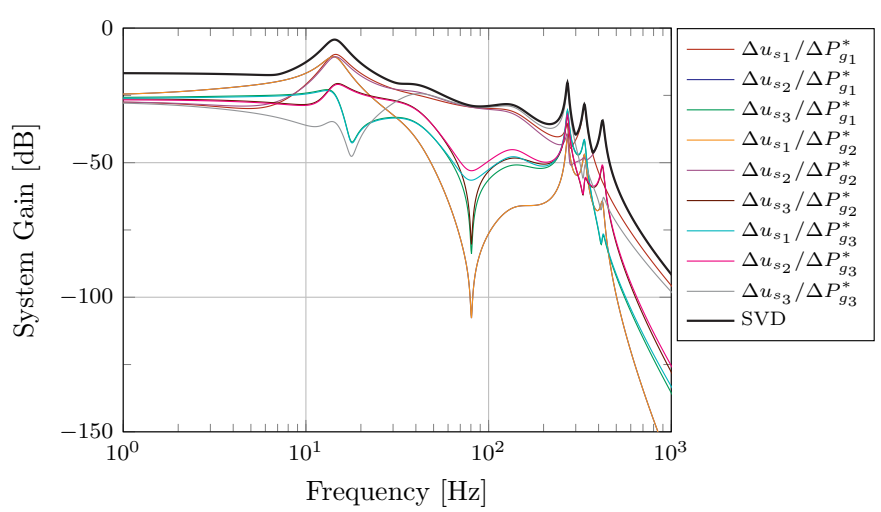

Figure 5. Comparison between the SVD representation and the 9 transfer functions of the 3-terminal MTDC system when the DC cables are modelled as fitted PI sections and for a voltage-droop parameter of $10 \%$.

The SVD representation corresponds to the maximum possible gain of the system, that is, the system gain in the most amplified direction of the output vector at any pulsation $\omega$. Hence, the maximum gain of the system is always bigger than the maximum of all the individual transfer function gains, as plotted in Figure 5. And since the maximum of the singular values depends on the root-mean-square of the DC voltage deviations $\Delta u_{s_{1}}, \Delta u_{s_{2}}$ and $\Delta u_{s_{3}}$, the SVD's shape follows the pattern of the transfer function with the biggest gain (especially when one of the outputs is more amplified than the others).

\section{Design of the Voltage-Droop Controller Gain}

\section{A. Voltage-Droop Parameter Selection}

The selection of the value of the voltage-droop parameter $k_{v}$ is crucial to ensuring the proper behaviour of the MTDC system since this parameter highly impacts the DC voltage level of the HVDC grid (both in transient and steady-state) and thus the conformity of the system to satisfy the maximum allowable DC voltage constraint of $\pm 5 \%$.

The impact of the voltage-droop parameter on the MTDC system can easily be assessed from a static point of view, as in [6] and [15], where a power reference modification
$\Delta P_{g}^{*}$ of one of the converters generates a computable DC voltage deviation. However, from a dynamic point of view, it is necessary to investigate how the voltage-droop parameter impacts the system when the latter is excited by oscillatory inputs like the output power of the WFC, or poorly-damped power oscillations generated by the $\mathrm{AC}$ grids.

\section{B. The DC Voltage Constraint}

The DC voltage constraint of $\pm 5 \%$ can be represented as a constant boundary and compared to the frequency response of the maximum singular values of the MTDC system. If the maximum singular values does not exceed this constraint boundary at any pulsation $\omega$, then the DC voltages of the three stations of the MTDC system are guaranteed to satisfy the maximum DC voltage constraint.

The maximum allowable power-reference deviation of the GSCs is assumed to be fixed at \pm 0.1 p.u. by the TSOs of their respective $\mathrm{AC}$ grids while the possible maximum power-reference deviation of the WFC is a change of \pm 0.2 p.u. This means that, for the frequency response of the DC voltage deviation output vector $y=\left[\begin{array}{lll}\Delta u_{s_{1}} & \Delta u_{s_{2}} & \Delta u_{s_{3}}\end{array}\right]^{T}$ with regards to the power-reference deviations input vector $u=\left[\begin{array}{lll}\Delta P_{g_{1}}^{*} & \Delta P_{g_{2}}^{*} & \Delta P_{g_{3}}^{*}\end{array}\right]^{T}$, the $\pm 5 \%$ maximum DC voltage constraint (i.e. \pm 0.05 p.u.) becomes:

$$
\begin{aligned}
& 20 \log _{10}\left(\frac{\sqrt{3 \cdot \Delta u_{s_{\max }}^{2}}}{\sqrt{\Delta P_{g_{1 \max }^{*}}^{*}+\Delta P_{g_{2 \max }^{*}}^{*}+\Delta P_{g_{3_{\max }}^{*}}^{2}}}\right) \\
= & 20 \log _{10}\left(\frac{\sqrt{3 \cdot(0.05)^{2}}}{\sqrt{\left(2 \cdot(0.1)^{2}+(0.2)^{2}\right)}}\right) \\
= & -9.0309 \mathrm{~dB}
\end{aligned}
$$

\section{SVD Analysis of the 3-Terminal HVDC System}

Figure 6 shows the maximum singular values $\bar{\sigma}(G)$ for different values of the voltage-droop parameter of the two GSCs' voltage-droop controller when the DC cables are modelled by a fitted PI section. In order to satisfy the $\pm 5 \%$ voltage deviation constraint, the maximum of the singular values must not bypass the constraint boundary computed in (5) and represented as the grey area of Figure 6.

All the tested values of the voltage-droop parameter allow the system to maintain the maximum voltage deviation within $\pm 5 \%$ of the nominal voltage value in steady state. This can be verified in Figure 6 as no frequency response of the MTDC system bypasses the constraint boundary in steady state, for any droop value. However, resonance peaks are observed at relatively low frequencies. These resonances can violate the maximum allowable DC voltage imposed on the MTDC system, especially if the wind-farm power reference (corresponding to the harvested wind power) excites the system at those frequencies.

For this specific 3-terminal MTDC system, the only voltagedroop parameter values $\left(k_{v}\right)$ that strictly satisfy the DC voltage constraint for any frequency are located between 0 and 8.8\% and then between 18.8 and $25 \%$. Some complementary studies 


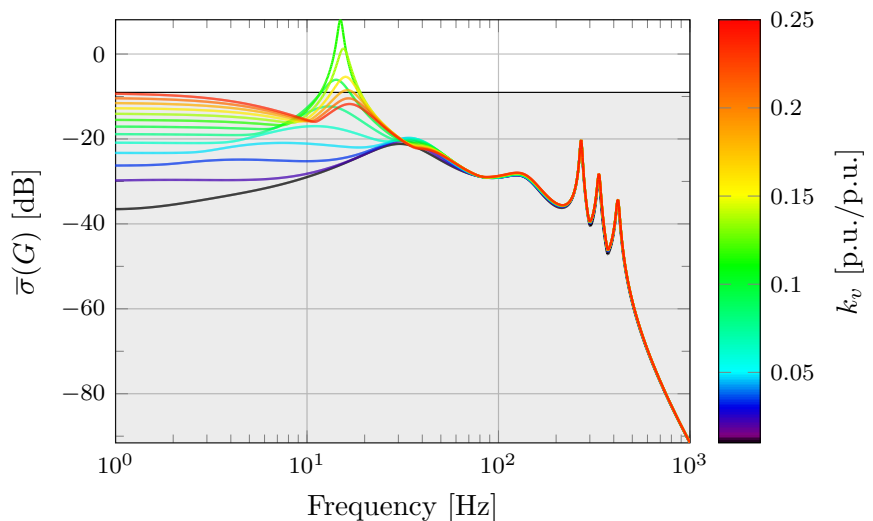

Figure 6. Maximum singular value of the DC voltage errors with regards to the power references of the converters for the 3-terminal MTDC system and for DC cables modelled as "fitted-PI" sections.

with different constraints, such as the maximum current amplitude in the IGBT of the converters, will then narrow further the possible range of the droop value [7], [9].

\section{Impact of the CABle Model on the Frequency AND TIME RESPONSES OF THE SYSTEM}

\section{A. Case of the 3-Terminal MTDC System}

Figure 7 shows the frequency response of the MTDC system for the four DC cable models, for voltage-droop parameter values $\left(k_{v}\right)$ of $1 \%, 3.4 \%, 6.3 \%, 9.6 \%, 13.6 \%$ and $18.2 \%$ (chosen arbitrarily).

In steady state, the four DC cable models have the same behaviour and satisfy the maximum allowable voltage-deviation constraint, which is consistent with the static study. At high and very high frequencies, the four models are also equivalent: the MTDC system shows 3 succeeding resonance peaks corresponding to the coupling between the DC cables and the inner current loops of the converters [13], and then acts as a low-pass filter.

However, from a dynamic point of view, it appears that the use of a $\mathrm{RC}$ section results in a single resonance peak around $70 \mathrm{~Hz}$ related to the dominant DC voltage mode of the system [13]. Similarly, the frequency response corresponding to the classical PI section presents a strong resonance peak around 15 $\mathrm{Hz}$ that always prevents the system from satisfying the voltage constraint. This resonance peak is still present but greatly attenuated with the fitted PI and the coupled PI sections, to the point that two separate ranges of voltage-droop parameter values satisfy the voltage constraint (] $0 ; 0.088[\cup] 0.188 ; 0.25[$ for the fitted PI and ]0;0.054[ $\cup] 0.097 ; 0.25[$ for the coupled PI).

The cable model influences the amplitude and the frequency of some of these dynamics. In fact, the use of a classical PI section can be misleading: the SVD with classical PI models implies that the system can never satisfy the constraint while the more accurate fitted PI and coupled PI models indicate the opposite, and even then, the range of acceptable voltagedroop parameter values differs from one model to another.
Hence, designing the voltage-droop parameter based on a system with DC cables modelled as classical PI sections leads to excessively conservative results.

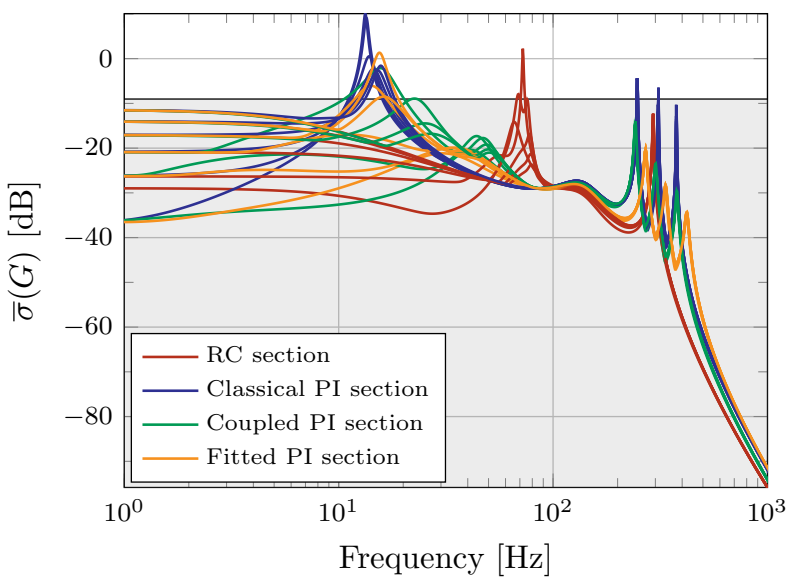

Figure 7. Maximum singular value of the DC voltage errors with regards to the power references of the converters for 4 different DC cable models and 6 different values of the voltage-droop parameter.

\section{B. EMT Simulations of the 3-Terminal MTDC System}

Figure 8 shows the time response of the DC voltage at the WFC terminals $\left(u_{s_{3}}\right)$ when the power reference of the WFC is subject to a -0.2 p.u. step, equivalent to the tripping of the offshore wind farm. This scenario is the most aggressive one in terms of time domain (step power deviation). The time response is plotted for four EMT simulations, corresponding respectively to the four DC cable models described in Section II-A.

When the DC cables are modelled as RC sections, the MTDC system as a whole acts like a low-pass filter and the voltage oscillations correspond to the DC voltage mode whose resonance peak was observed around $70 \mathrm{~Hz}$ in Figure 7. This simple cable model does not allow the full system to accurately illustrate the coupling between the DC cables and the control loops of the converters, and thus is not reliable when it comes to analysing the dynamic behaviour of the MTDC system.

The response of the system with DC cables modelled as classical PI sections shows oscillations at a frequency of 13.3 $\mathrm{Hz}$, corresponding to the first resonance peak observed in the singular value representation of Figure 7. This confirms the statement in [9], which is also using the classical PI cable model to obtain the state-space model of the MTDC system, that a DC oscillation damping loop was needed to prevent these oscillations.

However, the more precise fitted PI and coupled PI models of the DC cable showed an attenuated first resonance peak at $35 \mathrm{~Hz}$ and $50 \mathrm{~Hz}$ respectively in Figure 7, whose impact is almost not perceptible on the time response of the DC voltage of Figure 8, implying that the DC oscillation damping loop is actually not needed in this specific 3-terminal MTDC system configuration since these oscillations are generated by the limitations of the classical PI section representation of a 
DC cable, and are greatly attenuated with a more detailed cable model. It could, however, improve the performance of the system [9].

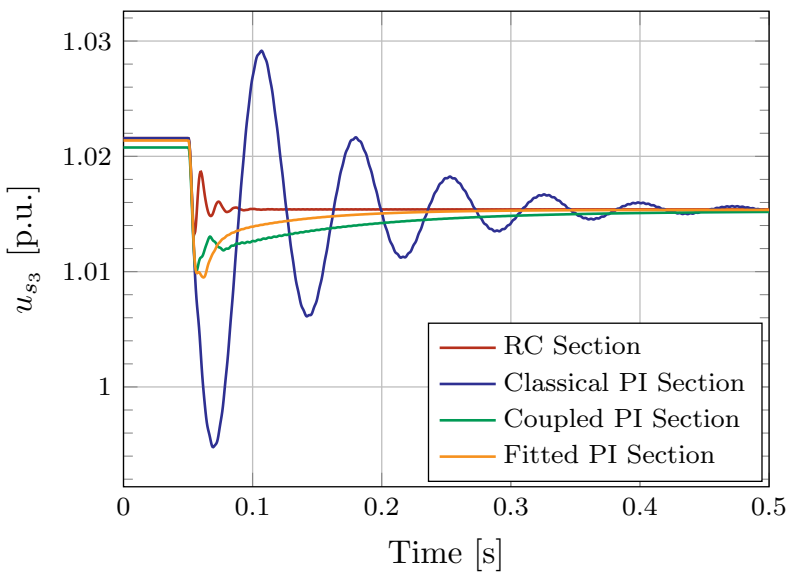

Figure 8. DC voltage at the WFC terminal for all four DC cable models, for a power reference step of -0.2 p.u. of the WFC $\left(k_{v}=5 \%\right)$.

\section{CONCLUSION}

The design of MTDC systems should ensure that the DC voltage does not exceed a maximum allowable DC voltagedeviation constraint. Hence, the choice of the voltage-droop parameter of the converters is extremely important because it impacts the DC voltage behaviour of the system. Yet, while the choice of the droop parameter from a static point of view can easily be achieved, its range must be narrowed even further in order for the system to also comply with the DC voltage constraint from a dynamic point of view.

An MTDC system is a MIMO plant. Thus, its input and output vectors have directions that greatly impact the gain of the system, making the study of the transfer functions inadequate. The frequency response analysis can still be performed thanks to the Singular Value Decomposition method, where the maximum of the singular values expresses the maximum possible gain of the system at any pulsation $\omega$.

The SVD representations of the system obtained with different DC cable models show that the classical PI section generally used to model the DC cables may not be an accurate representation of the dynamic behaviour of a DC cable and should not be used for SVD studies. The use of an improved PI section model of the DC cables shows that the system is naturally damped and that, for the very basic 3-terminal topology of the HVDC grid of this paper, two separate ranges of voltage-droop parameter values allow the system to comply with the DC voltage constraint.

The SVD tool can easily be computed for any system whose state-space representation is known. While this paper focused on a 3-terminal MTDC system with Voltage Source Converters, the next step is to replace the VSCs by the new generation of converters such as the Modular Multi-level Converters (MMC) as they will most likely be the most used converters for HVDC grids in the future and their dynamics are more versatile than for the VSCs.

\section{REFERENCES}

[1] International Energy Agency (IEA), "Wind energy technology roadmap." Report, 2013.

[2] N. Flourentzou, V. Agelidis, and G. Demetriades, "VSC-based HVDC power transmission systems: An overview," Power Electronics, IEEE Transactions on, vol. 24, pp. 592-602, March 2009.

[3] C. Dierckxsens, K. Srivastava, M. Reza, S. Cole, J. Beerten, and R. Belmans, "A distributed DC voltage control method for VSC MTDC systems," Electric Power Systems Research, vol. 82, no. 1, pp. 54 - 58, 2012.

[4] J. Beerten and R. Belmans, "Modeling and control of multi-terminal VSC HVDC systems," Energy Procedia, vol. 24, pp. 123-130, 2012.

[5] A. Egea-Alvarez, J. Beerten, D. Van Hertem, and O. Gomis-Bellmunt, "Primary and secondary power control of multiterminal HVDC grids," in $A C$ and DC Power Transmission (ACDC 2012), 10th IET International Conference on, pp. 1-6, Dec 2012.

[6] S. Akkari, M. Petit, J. Dai, and X. Guillaud, "Interaction between the voltage-droop and the frequency-droop control for multi-terminal HVDC systems," in AC and DC Power Transmission, 11th IET International Conference on, pp. 1-7, Feb 2015.

[7] E. Prieto-Araujo, F. Bianchi, A. Junyent-Ferré, and O. Gomis-Bellmunt, "Methodology for droop control dynamic analysis of multiterminal VSC-HVDC grids for offshore wind farms," Power Delivery, IEEE Transactions on, vol. 26, pp. 2476-2485, Oct 2011.

[8] R. Eriksson, J. Beerten, M. Ghandhari, and R. Belmans, "Optimizing DC voltage droop settings for AC/DC system interactions," Power Delivery, IEEE Transactions on, vol. 29, pp. 362-369, Feb 2014.

[9] E. Prieto-Araujo, A. Egea-Alvarez, S. Fekri, and O. Gomis-Bellmunt, "DC voltage droop control design for multi-terminal HVDC systems considering AC and DC grid dynamics," Power Delivery, IEEE Transactions on, vol. PP, no. 99, pp. 1-1, 2015.

[10] J. Beerten, S. D'Arco, and J. Suul, "Cable model order reduction for HVDC systems interoperability analysis," in $A C$ and $D C$ Power Transmission, 11th IET International Conference on, pp. 1-10, Feb 2015.

[11] O. Ramos-Leanos, J. Naredo, J. Mahseredjian, C. Dufour, J. GutierrezRobles, and I. Kocar, "A wideband line/cable model for real-time simulations of power system transients," Power Delivery, IEEE Transactions on, vol. 27, pp. 2211-2218, Oct 2012.

[12] P. Rault, Dynamic Modeling and Control of Multi-Terminal HVDC Grids. PhD thesis, Laboratory L2EP, University Lille Nord-de-France, 2014.

[13] S. Akkari, J. Dai, M. Petit, P. Rault, and X. Guillaud, "Small-signal modelling for in-depth modal analysis of an MTDC system," in Electrical Power and Energy Conference (EPEC), 2015 IEEE, pp. 233-238, Oct 2015.

[14] S. Skogestad and I. Postlethwaite, Multivariable Feedback Control, Analysis and Design. John Wiley \& Sons, 2007.

[15] S. Akkari, J. Dai, M. Petit, and X. Guillaud, "Coupling between the frequency droop and the voltage droop of an AC/DC converter in an MTDC system," in PowerTech, 2015 IEEE Eindhoven, pp. 1-6, June 2015.

\section{APPENDIX}

\section{A. Parameters of the Fitted PI Model of the DC Cable}

$$
\begin{array}{ll}
r_{z_{1}}=1.1724 \cdot 10^{-1} \Omega / \mathrm{km} & l_{z_{1}}=2.2851 \cdot 10^{-4} \mathrm{H} / \mathrm{km} \\
r_{z_{2}}=8.2072 \cdot 10^{-2} \Omega / \mathrm{km} & l_{z_{2}}=1.5522 \cdot 10^{-3} \mathrm{H} / \mathrm{km} \\
r_{z_{1}}=1.1946 \cdot 10^{-2} \Omega / \mathrm{km} & l_{z_{1}}=3.2942 \cdot 10^{-3} \mathrm{H} / \mathrm{km} \\
g_{c s}=7.6333 \cdot 10^{-11} \mathrm{~S} / \mathrm{km} & c_{c s}=1.9083 \cdot 10^{-7} \mathrm{~F} / \mathrm{km}
\end{array}
$$

B. Parameters of the Coupled PI Model of the DC Cable

$$
\begin{aligned}
& r_{c}=5.3466 \cdot 10^{-3} \Omega / \mathrm{km} \\
& l_{c}=3.7399 \cdot 10^{-3} \mathrm{H} / \mathrm{km} \\
& m_{c s}=3.5738 \cdot 10^{-3} \mathrm{H} / \mathrm{km} \\
& g_{c s}=6.2067 \cdot 10^{-8} \mathrm{~S} / \mathrm{km}
\end{aligned}
$$$$
r_{s}=3.3111 \cdot 10^{-2} \Omega / \mathrm{km}
$$$$
l_{s}=3.5726 \cdot 10^{-3} \mathrm{H} / \mathrm{km}
$$$$
c_{c s}=2.4696 \cdot 10^{-7} \mathrm{~F} / \mathrm{km}
$$

\section{Parameters of the VSCs}

$$
\begin{array}{lll}
U_{s_{b}}=640 \mathrm{kV} & S_{b}=1 \cdot 10^{9} \mathrm{VA} & \cos \varphi_{n}=1 \\
H=40 \mathrm{~ms} & C_{s}=195.31 \mu \mathrm{F} & k_{v_{\text {droop }}}=5 \%
\end{array}
$$

